Applied Remote Sensing

\title{
Subgrid-scale cirrus observed by lidar at mid-latitude: variability effects of the cloud optical depth
}

Philippe Keckhut Jean-Marie Perrin Gérard Thuillier Christophe Hoareau Jacques Porteneuve Nadège Montoux 


\title{
Subgrid-scale cirrus observed by lidar at mid-latitude: variability effects of the cloud optical depth
}

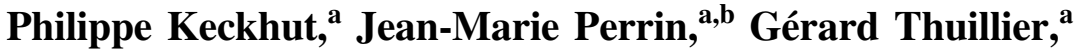 \\ Christophe Hoareau, ${ }^{a}$ Jacques Porteneuve, ${ }^{a}$ and Nadège Montoux ${ }^{c}$ \\ ${ }^{a}$ Versailles St-Quentin University, Pierre et Marie Curie University, \\ Laboratoire Atmosphères, Milieux, Observations Spatiales, CNRS/INSU, \\ UMR8190, 11, boulevard d'Alembert, 78280 Guyancourt, France \\ keckhut@latmos.ipsl.fr \\ bobservatoire de Haute Provence, PYTHEAS, 04870 Saint Michel l'Observatoire, France \\ ${ }^{c}$ Laboratoire de Météorologie Physique, Blaise Pascal University/CNRS/OPGC_UMR 6016, \\ les Cezeaux, 24 avenue des Landais, 63177 Aubière, France
}

\begin{abstract}
The temporal variability of the 532-nm optical depth of cirrus clouds observed with a lidar at Observatory of Haute-Provence $\left(43.9^{\circ} \mathrm{N}, 5.7^{\circ} \mathrm{E}\right.$, and $683-\mathrm{m}$ altitude), has been analyzed. While advection dominates at the first order, variability of the optical depth on timescales of minutes can be related to spatial fluctuations of cloud properties on typical scales of a few kilometers. Log-normal distributions of the optical depth have been used to model the variability of the cirrus optical depth as observed by lidars. These investigations have been performed for three independent classes of cirrus. The log-normal distribution of the optical depth is applicable to the classes of thin clouds; however, for thick clouds, likely due to successive freezing/defreezing effects, the distribution is rather bimodal. This work compares the effects of visible solar light scattered by inhomogeneous cirrus to effects generated by homogeneous clouds having a constant geometrical thickness using the short-scale lidar observations of optical depth distribution and an analytical approach. In the case of thin cirrus, the scattering of solar light reaching the ground is stronger for inhomogeneous than homogeneous cirrus. In case of thick cirrus, multiplescattering processes need to be considered. The conclusion is that log-normal distribution of the cirrus optical depth should be considered in any radiative calculation in case of model grids larger than a few kilometers whatever the cirrus type is. (C) The Authors. Published by SPIE under a Creative Commons Attribution 3.0 Unported License. Distribution or reproduction of this work in whole or in part requires full attribution of the original publication, including its DOI. [DOI: 10.1117/1.JRS.7.073530]
\end{abstract}

Keywords: cirrus; lidar; subgrid variability; cloud optical depth.

Paper 12078 received Apr. 6, 2012; revised manuscript received Mar. 18, 2013; accepted for publication Apr. 5, 2013; published online Jul. 30, 2013.

\section{Introduction}

\subsection{Cloudiness and Climate Modelling}

The processes controlling the distribution of clouds inducing radiative effects are key factors in climate modelling to determine the rate and magnitude of climate changes. The cloud radiative force is still one of the major uncertainties for climate modeling as pointed out by the Intergovernmental Panel on Climate Change (IPCC) report. ${ }^{1}$ As a consequence of the difficulty to correctly model the cloud effects, numerical climate simulations exhibit large discrepancies in terms of predicted mean temperature changes. ${ }^{2}$

Prognostic cloud schemes explicitly compute the average concentrations of water and ice in the cloudy part of each spatial resolution element (or grid cell), typically the boxes of few degrees in latitude and longitude. However, the large variability of water vapor covering almost all relevant atmospheric spatial and temporal scales is the main origin of cirrus inhomogeneity and consequently in climate modelling difficulties, as these models use mean gridded values and do 
not take into account the subgrid scale variability. ${ }^{3}$ This study is an attempt to derive information about the subgrid variability of the cirrus optical depth using lidar measurements.

\subsection{Cirrus Variability Observed by Satellite}

Similarly, cloud properties deduced from satellite measurements may be biased because of various factors like three-dimensional (3-D) radiative effects resulting from cloud nonhomogeneities. ${ }^{4}$ Cirrus clouds, formed under the cold conditions of the upper troposphere, occur in an area where the horizontal transport is important even for tropics, when the convection locally contributes to an intense vertical transport of the water vapor. This adiabatic transport over quasihorizontal isentropes generates filamentary structures over a wide range of scales. A recent investigation ${ }^{5}$ using a high-resolution advection model has forecasted local water vapor concentrations of nearly $30 \mathrm{ppmv}$ at around 200 to $250 \mathrm{hPa}$, within filamentary structures with typical sections of 10 to $100 \mathrm{~km}$. The horizontal average over a box of several degrees will drastically reduce the water vapor gradient to the background value of around 15 to $20 \mathrm{ppmv}$ which is close to climatological values and sometimes remains below the threshold of cirrus cloud formation.

Passive methods aboard nadir satellites viewing, even if their footprint resolution increases nowadays, do not capture all cirrus types, ${ }^{6,7}$ especially those with optical depths smaller than 0.03 that are visually not detected from ground and are named as subvisible clouds. ${ }^{8}$ The limbscanning method allows a better detection of thin cirrus as their path of sight crosses the cirrus over a typical distance of $100 \mathrm{~km} .{ }^{9,10}$ Active methods using space and ground-based lidars provide data in good agreement, ${ }^{11,12}$ however, the orbit and speed of the satellite do not allow a detailed analysis of the short-term variability.

\subsection{Scattering in Clouds}

The World Meteorological Organization ${ }^{13}$ defines cirrus clouds as silky sheen or fibrous appearing as a clone's heritage of the classification made by Hildebrandson. ${ }^{14}$ Due to their altitude of occurrence of usually $>7 \mathrm{~km}$, cirrus cloud composition is dominated by ice crystals while the majority of clouds are composed of water droplets. For clouds that are mainly composed of ice, the main cause of the extinction of light is the scattering effect. ${ }^{15}$

Due to either fluctuations of the depth of the cirrus clouds or particle concentrations within the cirrus, the optical depth can be $<0.03$ or $>0.1$. Consequently, the scattered solar light by cirrus as received on the ground is due to single scattering processes or can include multiple-scattering contributions depending on the optical depth value. ${ }^{16}$ However, the amplitude of this effect strongly depends on the field of view collecting the scattered light. Lidars using small field of views ( $<5$ arc min) are less sensitive to this effect than instruments, which receive light at ground from all directions.

During the last decades, many experimental campaigns using complementary techniques have significantly improved the description of cloud particles and have revealed their large diversity in particle shape and size distribution. ${ }^{17}$ However, the exact link between microphysic cloud parameters associated with their formation processes and their radiative effects at the model gridscale have not provided the expected full physical parameters set as inputs to the numerical climate models. At the macroscopic scale, spatial inhomogeneity of clouds is also a possible candidate to explain the observed discrepancies between the model results of the radiative impact of a cloudy scene depending on the horizontal resolution and results using cirrus optical depths estimated from satellites.

The atmospheric water vapor transport either by convection and advection or through crystal sedimentation modifies the distribution of water vapor and ice particles concentration through highly nonlinear processes. Consequently, the description of cirrus occurrence using mean values is also biased due to the subgrid scale variability. ${ }^{18} \mathrm{Gu}$ and Liou ${ }^{19}$ have tested the radiative effects of broken clouds and multilayer clouds using a 3-D radiative transfer model. They show that cirrus clouds with a maximum overlap tend to produce less shortwave heating than those with random overlap, and furthermore, they show that broken clouds generate more shortwave heating and more infrared (IR) cooling compared to continuous cloud fields used in the simulation. 


\subsection{Lidar Measurements}

The magnitude of the radiative forcing by cirrus depends on its altitude and their optical depth, which is a function of both the geometrical and optical properties of the particles and their number concentration..$^{20,21}$ For a given cirrus cloud, those parameters vary over the three spatial dimensions due to small-scale water vapor structures and variability in time. Despite the difficulty to deduce microphysical properties from lidar measurements, ${ }^{22}$ the vertical structure, as well as its temporal evolution associated with air masses passing over a given location, is well described by a ground-based lidar.

The short-wave radiative transfer effects through cirrus can be calculated from the optical depth $^{23}$ and their nonhomogeneity can be represented by optical depth distribution functions. Kärcher and Strom ${ }^{24}$ suggest that dynamics are a key factor for cirrus formation and, consequently, for optical depth distribution. Kay et al. ${ }^{25}$ investigate optical depth distributions based on dynamical processes. They show that these distributions primarily depend on the ice crystal fallout time scale and that the vertical wind velocity is a key factor in these processes. Lidars also allow probing cirrus structure and deriving their optical depth with vertical and temporal resolutions (see Sec. 4). Combined measurements at two wavelengths ${ }^{22}$ such as lidar and mid-infrared radiometry measurements, to obtain the cloud optical depth distribution and to estimate solar albedo, show biases as large as $25 \%$.

The routine measurements performed at Observatory of Haute-Provence $\left(43.90^{\circ} \mathrm{N}\right.$ and $5.71^{\circ} \mathrm{E}$ ) during nighttime since 1997 have permitted usb to produce a first lidar climatology based on the morphologic cloud parameters including mean altitude, geometrical and optical depth. ${ }^{26}$ During the 1997 to 1999 period, most of the measurements were conducted during night time. Since 1999, lidar measurements are carried out around three times a week at nighttime. The cluster analysis ${ }^{27}$ including mean cloud temperatures and cloud vertical distributions, has revealed that three statistically distinct classes can be identified. Each class corresponds to different formation processes permitting one to rank it based upon morphological parameters. The class attribution is not yet fully demonstrated as in addition to standard large-scale cirrus, contrail-induced cirrus and isentropic filamentary from tropical areas can be the two other mechanisms of cirrus occurrence at mid-latitudes. Cirrus are observed in the vicinity of the tropopause corresponding to sporadic clouds in filamentary of wet air masses advected from the tropics ${ }^{28}$ and have been reproduced using a high-resolution advection model generating a high concentration water vapor field with realistic structures. ${ }^{5}$

The power of the lidar and the low background light allow accessing the high-altitude clouds with high sampling rate on the order of minutes. The variability of the optical depth derived from lidar measurements can be related to horizontal cloud variability generated by advection.

\subsection{Present Study}

In this work, it is proposed to investigate the radiative effect of cirrus of the different classes already identified ${ }^{27}$ having a log-normal distributed optical depth. For that, we compare the impact of the inhomogeneous cirrus optical depth on ground irradiance with the impact of homogeneous cirrus optical depth equal to the most probable one of the log-normal distribution.

Section 2 describes the lidar, whereas Sec. 3 is dedicated to the method for derivation the cirrus optical depth. Section 4 provides us with the short-term ( $2 \mathrm{~min}$ and $40 \mathrm{~s})$ distribution of the optical depth and Sec. 5 presents the analytical calculation of the optical depth and results as a function of cirrus class. A conclusion is given in Sec. 6.

\section{Lidar Description}

The lidar, which operates at Observatory of Haute-Provence in South of France was initially designed to measure Rayleigh scattering to derive temperature ${ }^{29}$ as well as stratospheric aero$\operatorname{sols}^{30}$ in the framework of the Network for the Detection of Stratospheric Changes, is described in Ref. 31. In 1994, three channels were added for water vapor, nitrogen density, and aerosols for simultaneous cirrus retrieval. The system is based on the second harmonic of a pulsed Nd:Yag laser, which emits pulses of $300 \mathrm{~mJ}$ output energy at $532 \mathrm{~nm}$ with $50 \mathrm{~Hz}$ repetition rate. 
Backscattered photons are collected into different channels by means of optical fibers mounted in the focal plane of mirrors having different sizes and in a mosaic of four mirrors for the Rayleigh channels. The lidar transmitter-receiver system is shown in Fig. 1. The cirrus channel used a $20-\mathrm{cm}$ telescope and an optical fiber of $1-\mathrm{mm}$ diameter. The field of view is equal to $1 \mathrm{mrad}$. Because the measurements were also dedicated to stratospheric aerosol analyses, an electronic shutter system has been added to reduce the noise induced by the initial light pulse. Due to this electronic shutter, clouds are only detected $>6 \mathrm{~km}$ height. The photon counting system has a $0.5 \mathrm{~ms}$ bin width, which corresponds to a height resolution of $75 \mathrm{~m}$.

The cirrus detection system (Fig. 2), connected to the receiver by the optical fibers, is composed of a primary lens that provides a collimated beam followed by an interference filter. A mechanical electric shutter is mounted after the filter to adjust the number of photons to be received on the photocathode of the photomultiplier. After the shutter, a final lens is used to focus the photons on the photocathode. The adjustment of the photon flux is required because the lidar signal due to the presence of clouds may vary to a large extent. This device allows us to optimize the lidar signal and the quality of the retrieval. The detector is operated in a photocounting mode. To avoid specular reflections for normal incidences that occur in some cases when ice crystals are horizontally oriented, ${ }^{31-34}$ the lidar beam is tilted from zenith by a few degrees.

\section{Lidar Data Analysis}

Lidar data obtained at Observatory of Haute-Provence have been analyzed using a method similar to that described by Goldfarb et al. ${ }^{26}$ Lidars provide accurate and direct data for cloud heights

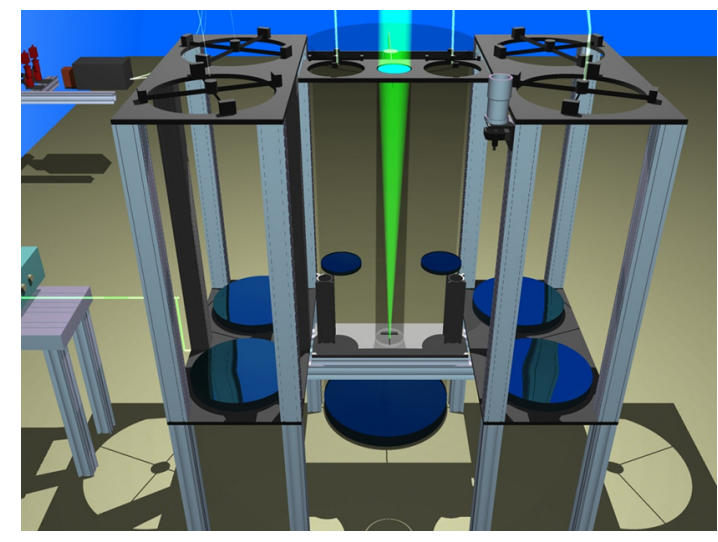

Fig. 1 Synthetic view of the emission-reception system of the observatory of Haute-Provence (OHP) aerosol-temperature lidar.

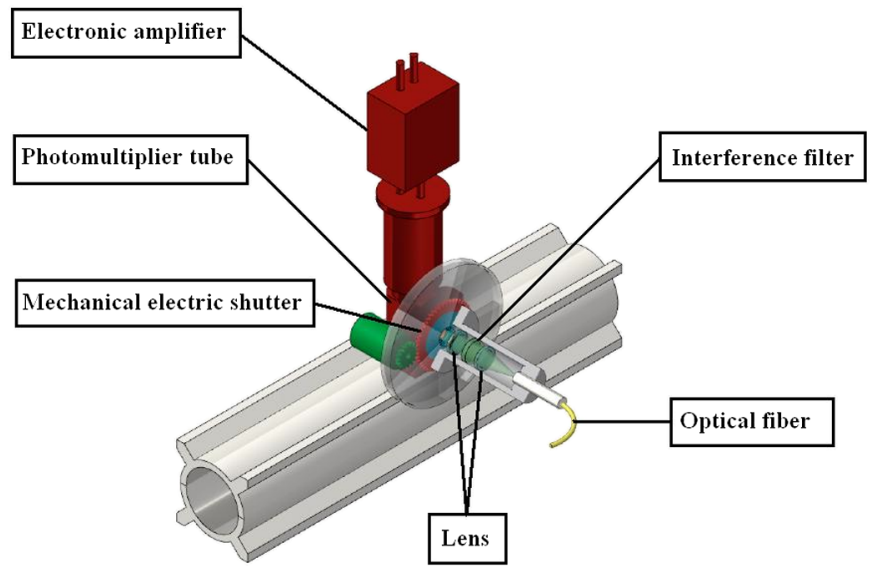

Fig. 2 Optical system for filtering and detection of the aerosols-cirrus channel of the OHP lidar. 
and vertical thickness as well as useful information about the vertical distribution of light scattered inside the clouds. However, due to the large variability of the vertical shape of clouds, we choose to investigate cloud structure through a more integrative parameter, namely the optical depth. The lidar retrieval of the cirrus optical depth requires some assumptions such as the lidar ratio (LR) corresponding to the extinction-backscattering ratio.

The optical depth is calculated from the air density $n_{\text {air }}(z)$, the scattering ratio $[\operatorname{SR}(z)]$, as a function of altitude $z$ (from sea level), the Rayleigh backscattering cross section $\left(\sigma_{\text {Rayleigh }}\right)$, and the LR, using the relationship:

$$
\tau=(\mathrm{LR}) \sigma_{\text {Rayleigh }} \int_{Z_{\min }}^{Z_{\max }} n_{\text {air }}(z)[\operatorname{SR}(z)-1] \mathrm{d} z .
$$

The scattering ratio is calculated from the elastic-backscatter lidar signal at $532 \mathrm{~nm}$ due to molecules and particles and the Nitrogen Raman scattering with a shifted wavelength (at $607 \mathrm{~nm}$ ) proportional to air density. ${ }^{35}$ The scattering ratio is calibrated in the altitude range from 20 to $25 \mathrm{~km}$ where we assume aerosol-free conditions. During the preceding years, no major volcanic eruption occurred and consequently, the background stratospheric aerosol concentration is very low. The optical depth is calculated for the altitude range from 7 to $14 \mathrm{~km}$ where most of the cirrus clouds were observed..$^{26}$ The LR can be directly derived from the lidar extinction profile and backscatter coefficient, however, large uncertainties and biases concerning the retrieved values are likely obtained ${ }^{35}$ in case of subvisible cirrus and thick clouds (when no Rayleigh scattering above the cloud is detected). The LR depends on the optical characteristics of the cirrus particles including size, shape, and particle composition and thus can vary vertically within a cloud. Usually, the value of the LR is assumed constant within the cloud while we know that bigger particles are found at the bottom of the cloud and smaller particles at the top. The attempts to systematically retrieve LR directly using the lidar signal itself have been obtained with large uncertainties leading to a wide spread of values. ${ }^{36}$ The laser light is attenuated during its passage through the cloud. This is detectable on the lidar signals, however, for subvisible clouds, the attenuation is low and in that case, the optical depth is retrieved with large uncertainties. This is especially true for short integration times such as those we used in this study with initial averaging of $160 \mathrm{~s}$. For this reason, in the previous studies, the LR was set to a constant value of $18.2 \mathrm{sr}^{37}$ Recent studies report mean values of $30 \mathrm{sr}^{38,39}$ Most of these past investigations have been performed for optically thick cirrus and it was not demonstrated at that time that the cirrus clouds of different types have different LRs. Chen et al. ${ }^{40}$ show that the LR varies with the cirrus optical thickness. Subvisible clouds have LR around $20 \mathrm{sr}$ close to what have been used in previous studies ${ }^{26,39}$ and show values larger than $30 \mathrm{sr}$ for thicker clouds. To derive optical depth distributions, the latter two values were used according to the cirrus classes. This is why, in this study, optical depths for both classes corresponding to thin clouds (C1 and C3) were retrieved using the Platt's value, and for thicker clouds (class C2) have used a larger value of $30 \mathrm{sr}$. The air density number concentration $n_{\text {air }}(z)$ is calculated from the Mass Spectrometer Incoherent Scatter-Extented atmospheric model of 1990 (MSISE-90).

Because of the uncertainty mainly affecting the LR, the absolute value of the mean optical depth is likely obtained with a confidence of around 30\%. However, in this study, the main objective is the investigation of the shape of the optical depth distribution rather than the absolute value. However, we note that the LR retrieval method could induce uncertainty on the shape of the distribution itself. If we assume that the LR within a cirrus class is kept constant, the variability of the optical depth (and consequently the shape of the distribution) is only due to the variation of the particle number density. The uncertainly in the shape of the optical depth distribution will be discussed in Sec. 4.

Elementary measurements are profiles of the collected laser light backscattered by cirrus ice particles with integration time of 8000 shots and laser repetition rate of $50 \mathrm{~Hz}$ corresponding to an averaging time of $160 \mathrm{~s}$. Each set of measurements is composed of a temporal series of elementary measurements during several hours of observations. Usually, lidar measurements are averaged over a night to improve the SNR, which decreases with increasing altitude.

However, as shown in Fig. 3, long signal integration times tend to erase the temporal information (left panels) and can lead to confusion because single-thin clouds moving vertically may 


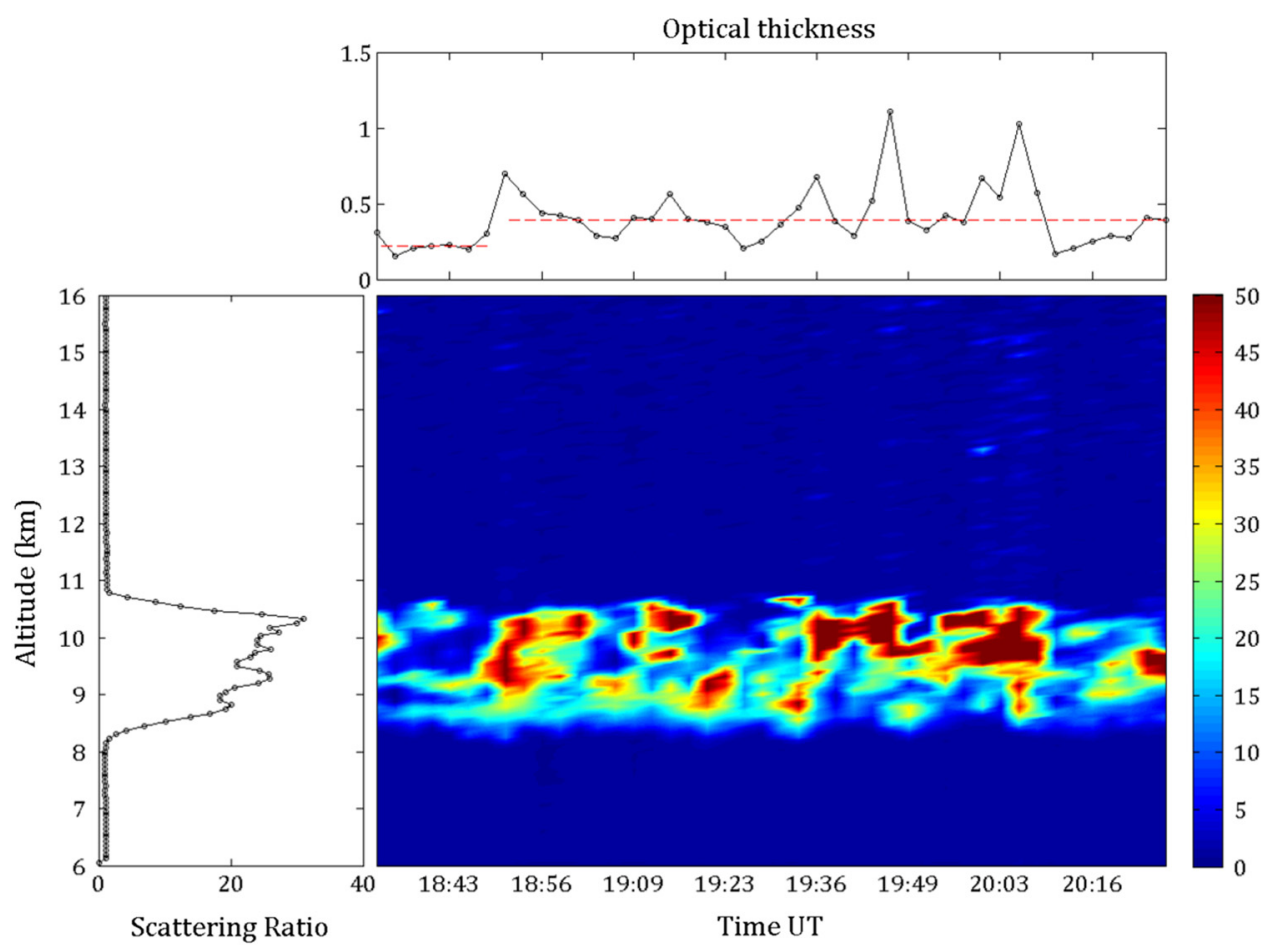

(a)

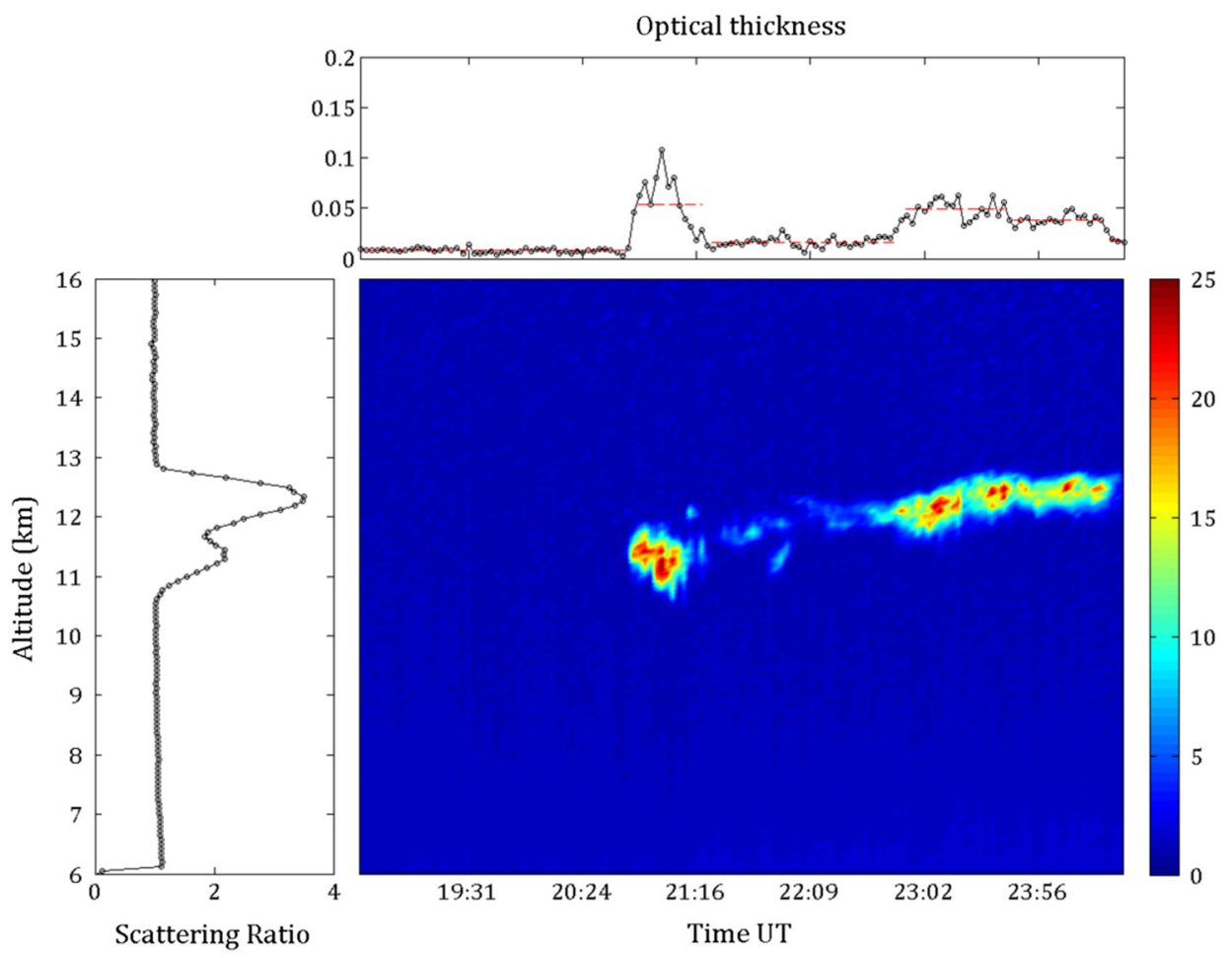

(b)

Fig. 3 Examples of scattering ratio evolution observed at $532 \mathrm{~nm}$ with lidar during two cirrus events. Scale is described with color legend ( 1 indicates no aerosol). Top panels present the average optical depth time evolution for the altitude range from 7 to $14 \mathrm{~km}$. Left panels correspond to profiles of the scattering ratio for the whole time period. Measurements have been performed on October 3 (a) and September 27, 2000 (b), respectively. 
be detected as a single deeper cloud or a multilayer cloud. Due to this possible altitude shift, it is not straightforward to follow the same air masses. For thin cirrus clouds, we have investigated the temporal evolution of their optical thickness estimated from the $160 \mathrm{~s}$ raw lidar backscatter signal averaged over the altitude range from 7 to $14 \mathrm{~km}$ (Fig. 3, upper panels). If a mean wind of $10 \mathrm{~m} / \mathrm{s}$ is assumed, then during $160 \mathrm{~s}$, a horizontal path of an air parcel of about $1.6 \mathrm{~km}$ is probed. If we consider the advection in the upper troposphere to be faster than cirrus formation, the temporal cloud structure observed can be considered to have an average of 1.6-km resolution.

\section{Variability of Cirrus Optical Depth: Observations}

The distribution of the optical depth deduced from each elementary measurement [quantities shown in Fig. 3(a)] gathered during a year (around 100 nights or $500 \mathrm{~h}$ of measurements) is shown in Fig. 4 on a log scale. Two maxima of the log distribution are observed corresponding to optical depth of 0.06 and 0.0006 , respectively. The optical depth distribution is fitted with two log-normal functions. While the uncertainty associated with the shape of the optical depth distribution can be affected by some retrieval assumptions $(L R, \ldots)$, the log-normal fits of the both modes indicate a satisfactory agreement with local differences from observations and shape models at the maximum equal to $25 \%$. In this study, it is assumed that a large part of the variability is probably due to the cirrus cloud types and then investigations were mainly focused on each of the three classes already identified. Because the properties of the cirrus clouds may depend on different formation processes, their variability should be studied for each class. The classification of cirrus types previously performed was based on morphological parameters provided by lidar observations (vertical depth and mean altitude), and external conditions given by the co-located radiosondes (temperature and tropopause height). Three classes ${ }^{27}$ were obtained. Table 1 shows that the variation of geometric thickness with altitude and the mean

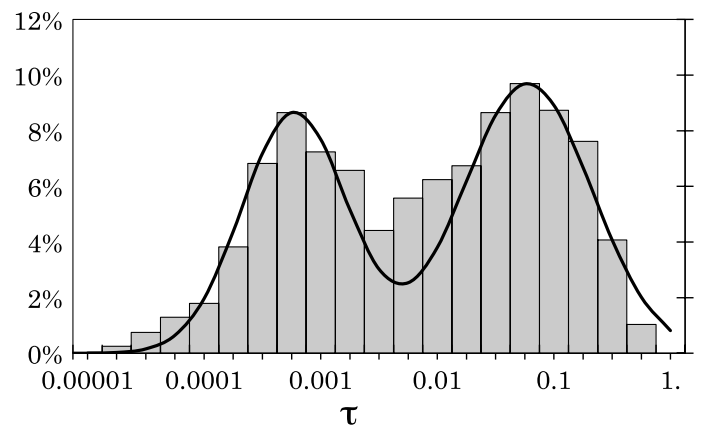

Fig. 4 Probability density distribution of the optical depth $(\tau)$ for all $160 \mathrm{~s}$ integration files obtained during the year 2000.

Table 1 Cirrus classes characteristics as a function of their morphology and variability distribution.

\begin{tabular}{lcccc}
\hline \hline $\begin{array}{l}\text { Cirrus } \\
\text { class }\end{array}$ & $\begin{array}{c}\text { Mean } \\
\text { Altitude } \\
(\mathrm{km})\end{array}$ & $\begin{array}{c}\text { Mean } \\
\text { Thickness } \\
(\mathrm{km})\end{array}$ & $\begin{array}{c}\text { Standard } \\
\text { deviation }\end{array}$ & $\begin{array}{c}\text { Most } \\
\text { probable } \\
\text { optical depth }\end{array}$ \\
\hline $\mathrm{C} 1$ & 8.6 & 0.9 & 0.45 & 0.032 \\
$\mathrm{C}{ }^{\prime}$ & 9.8 & 3.2 & 0.50 & 0.126 \\
$\mathrm{C}{ }^{\prime \prime}$ & 9.8 & 3.2 & 0.30 & 0.016 \\
$\mathrm{C} 3$ & 11.5 & 0.9 & 0.50 & 0.01 \\
\hline \hline
\end{tabular}


cloud altitudes are the strongest discriminant parameters. Histograms have been derived with data characteristic of each specific cluster (Fig. 5) and fitted by a log-normal function given the most probable value and the standard deviation of the distribution as shown hereafter:

$$
f(\log \tau)=f_{\max } \exp \left[-\frac{\left(\log \tau-\log \tau_{0}\right)^{2}}{2 \sigma_{0}^{2}}\right]
$$

where

(1) $f(\log \tau) d \log \tau$ is the frequency of the measured optical depth in the range $\log \tau$ to $\log \tau+d \log \tau$,

(2) $f_{\max }$ is the highest value of $f$ obtained when $\tau=\tau_{0}$,

(3) $\tau_{0}$ is the most frequently observed optical depth and $\sigma_{0}$ is its log decimal standard deviation.

From the Raman Nitrogen channel data, the air density profile is known, allowing us to retrieve the clouds and aerosols optical depth. The most probable value obtained around optical depth of 0.0006 exists for all the three classes (Fig. 5) with a standard deviation in the log scale

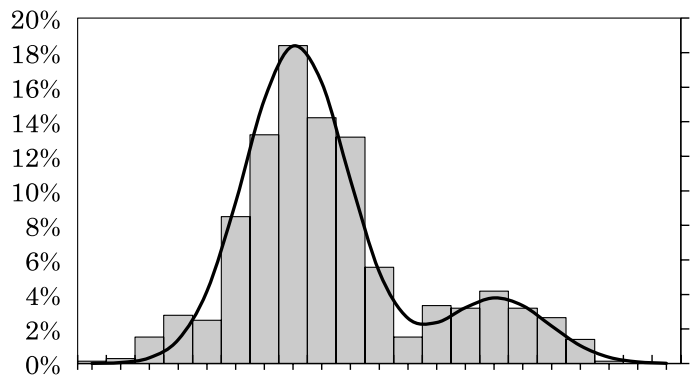

(a)

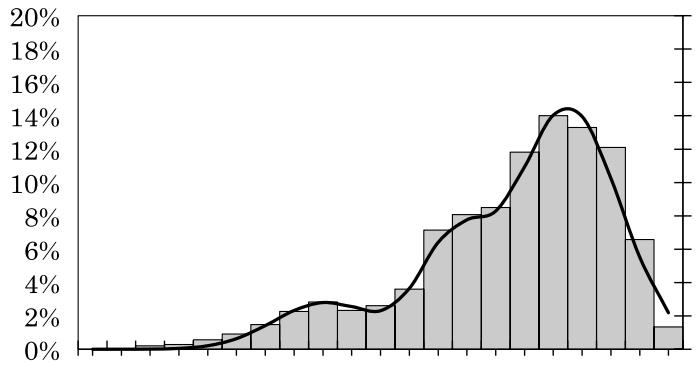

(b)

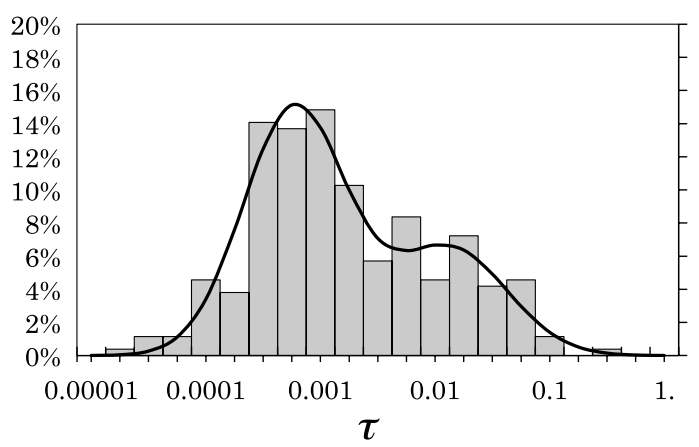

(c)

Fig. 5 Probability density of the optical depth $(\tau)$ for all the $160 \mathrm{~s}$ integration files obtained during the year 2000 for the first class corresponding to (a) thin low clouds (C3), (b) thick clouds (C2), and (c) thin clouds at the vicinity of the tropopause (C1). 
$\sigma_{0}$ of 0.45 . When cirrus clouds disappear within a night of observation, the measurements are related to the background aerosols. However, this background optical depth can also be due to the processing methodology. Indeed, to subtract the Rayleigh backscattered contribution of atmospheric molecules, Nitrogen Raman signal is used, however, its weakness adds some noise to the backscattered signal by cirrus and atmospheric aerosols. Because elastic and Raman scattering signals are simultaneously obtained, it does not include any bias. Moreover, to calibrate the molecular measurements, data are gathered at an altitude range that is expected to be free of aerosols. If this is not verified, a systematic bias is introduced as also observed for Caliop experiment. ${ }^{41}$ Furthermore, noise extraction associated with signal induces uncertainties.

The histogram of the first class of cirrus (Table 1) corresponding to low thin clouds reveals that the second maximum associated with the background remains with the same distribution [Fig. 5(a)]. The other class centered at $\tau=0.03[\log (\tau)=-1.5$ with a standard deviation of 0.45 in $\log$ scale] corresponds to cirrus observed at a mean altitude of $8.6 \mathrm{~km}$ and having a geometrical depth of $0.9 \mathrm{~km}$. During the night, this type of cirrus appears to be intermittent and a large part of the night is cloud free explaining the large pick associated to background conditions or noise $[\log (\tau)=-3.25$ with a standard deviation of 0.45]. It corresponds to an altitude where air is close to saturation with ice presence. ${ }^{42}$ The log-normal functions used as a fit reveal a better agreement for this class with a deviation $<10 \%$.

The histogram of the second cluster is a more complex one to model because it does not correspond to a pure log-normal distribution [Fig. 5(b)], suggesting two C2 subclasses. In addition to the background mode, the second maximum can be modeled by two log-normal functions with the main one centered on $\tau=0.13[\log (\tau)=-0.9]$ with a standard deviation of 0.45 (called hereafter $\mathrm{C} 2 /$ subtype) and the second one showing a maximum at $\tau=0.016[\log (\tau)=-1.8]$ with a standard deviation of 0.3 (called hereafter $\mathrm{C} 2$ " subtype)

The $\mathrm{C} 2$ class corresponds to clouds located at a mean height of $9.8 \mathrm{~km}$ and a mean geometrical thickness of $3.2 \mathrm{~km}$. Contrary to the two other cirrus classes, this one corresponds to more persistent clouds lasting several hours. This bimodal behavior was not expected to describe a single cirrus class. However, it could be associated with an oscillation between two modes as described by Kay et al. ${ }^{25}$ Using an adiabatic parcel model with explicit binned ice microphysics, Kay et al. ${ }^{25}$ simulate cirrus cloud optical depth distribution. They found that the distribution shape depends primarily on the ratio of the ice crystal fallout velocity to the timescales of other microphysical and dynamical processes that mainly form in two regimes. One corresponds to a dominant fallout regime for which the distribution exhibits a peak at large optical depth. The other one corresponds to a small fallout regime that exhibits a bimodal distribution due to multiple successive freezing events. Our observations of $\mathrm{C} 2$ cirrus type cluster could be like the succession of freezing/thawing effects as illustrated in the case presented in Fig. 3(a).

For the third class [Fig. 5(c)], the background mode is also detected, whereas another mode can be seen with a maximum around $\tau=0.01[\log (\tau)=-2$ with a standard deviation of 0.5$]$. This last class corresponds to clouds located at a mean height of $11.5 \mathrm{~km}$ with a mean geometrical depth of $0.9 \mathrm{~km}$. It is associated with dry filamentary structures isentropically transported from the tropical troposphere to the tropopause region at mid latitude as already observed ${ }^{13}$ and modeled by Ref. 14 . The deviation to the fit is larger than for $\mathrm{C} 1(10 \%)$ mainly because the sample is smaller than for the other cases. Those thin cirrus clouds located close to the tropopause are filamentary structured and are consequently observed sporadically. This could explain the smaller sampling and the relatively larger mode associated with background conditions when clouds are not observed.

\section{Estimation of the Horizontally Variable Optical Thickness}

The previous section shows that optical depth measurements are distributed with a log-normal function except the $\mathrm{C} 2$ class that is composed of two distributions. Then the calculation of the radiative effect on ground is expected to be different from the case of a homogeneous layer whose optical depth is generally the most probable one. Here, we calculate an estimate of the impact of such inhomogeneity on visible scattered solar light at ground. To this end, we 
quantify this impact using the analytical expressions of the average value of the optical thickness $\bar{\tau}$ from the log-normal function derived from lidar data and we compare $\bar{\tau}$ with $\tau_{0}$.

Let us consider a class of cirrus for which the value of the optical depth is included between $\tau_{i}$ and $\tau_{j}$. By definition and using Eq. (2) we have

$$
\bar{\tau}=\frac{1}{N_{C}} \int_{\log \tau_{i}}^{\log \tau_{f}} \tau f_{\max } \exp \left[-\frac{\left(\log \tau-\log \tau_{0}\right)^{2}}{2 \sigma_{0}^{2}}\right] d \log \tau,
$$

where $\begin{aligned} & \tau_{i} \geq 0 \\ & \tau_{i}<\tau_{0}<\tau_{f},\end{aligned}$ and $N_{C}$ is the normalization constant:

$$
N_{C}=\int_{\log \tau_{i}}^{\log \tau_{f}} f_{\max } \exp \left[-\frac{\left(\log \tau-\log \tau_{0}\right)^{2}}{2 \sigma_{0}^{2}}\right] d \log \tau .
$$

As $d \log \tau=1 / \operatorname{In} 10 d \tau / \tau$, then

$$
\bar{\tau}=\frac{f_{\max }}{N_{C} \operatorname{In} 10} \int_{\tau_{i}}^{\tau_{f}} \exp \left[-\frac{\left(\operatorname{In} \tau-\operatorname{In} \tau_{0}\right)^{2}}{2\left(\sigma_{0} \operatorname{In} 10\right)^{2}}\right] \mathrm{d} \tau .
$$

To calculate this integral, let

$$
x=\frac{\operatorname{In} \tau-\operatorname{In} \tau_{0}}{\sqrt{2 \sigma_{0} \operatorname{In} 10}} \quad \text { then } \tau=\tau_{0} e^{x \sqrt{2 \sigma_{0}} \operatorname{In} 10}
$$

and

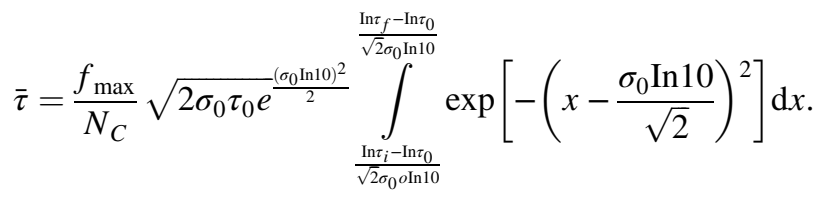

As $\tau_{i}=0$ if we let $u=x-\frac{\sigma_{0} \text { In10 }}{\sqrt{2}}$ we obtain

$$
\bar{\tau}=\frac{f_{\max }}{N_{C}} \sqrt{2} \sigma_{0} \tau_{0} e^{\frac{\left(\sigma_{0} \operatorname{In} 10\right)^{2}}{2}} \int_{-\infty}^{\frac{\operatorname{In} \tau_{f}-\operatorname{In} \tau_{0}}{\sqrt{2} \sigma_{0} \operatorname{In} 10}-\frac{\sigma_{0} \operatorname{In} 10}{\sqrt{2}}} e^{-u^{2}} \mathrm{~d} u .
$$

As by definition ${ }^{43,44}$

$$
\begin{gathered}
\int_{0}^{z} e^{-t^{2}} \mathrm{~d} t=\frac{\sqrt{\pi}}{2} \operatorname{erf}(z) \\
\bar{\tau}=\frac{f_{\max }}{N_{C}} \sigma_{0} \tau_{0} e^{\frac{\left(\sigma_{0} \operatorname{In} 10\right)^{2}}{2}} \sqrt{\frac{\pi}{2}\left[1+\operatorname{erf}\left(\frac{\operatorname{In} \tau_{f}-\operatorname{In} \tau_{0}}{\sqrt{2} \sigma_{0} \operatorname{In} 10}-\frac{\sigma_{0} \operatorname{In} 10}{\sqrt{2}}\right)\right] .}
\end{gathered}
$$

Using the same method, we obtain

$$
N_{C}=f_{\max } \sigma_{0} \sqrt{\frac{\pi}{2}}\left[1+\operatorname{erf}\left(\frac{\operatorname{In} \tau_{f}-\operatorname{In} \tau_{0}}{\sqrt{2} \sigma_{0} \operatorname{In} 10}\right)\right]
$$

Finally, 


$$
\bar{\tau}=\tau_{0} \exp \left[\frac{1}{2}\left(\sigma_{0} \operatorname{In} 10\right)^{2}\right] \frac{1+\operatorname{erf}\left(\frac{\operatorname{In} \tau_{f}-\operatorname{In} \tau_{0}}{\sqrt{2} \sigma_{0} \operatorname{In} 10}-\frac{\sigma_{0} \operatorname{In} 10}{\sqrt{2}}\right)}{1+\operatorname{erf}\left(\frac{\operatorname{In} \tau_{f}-\operatorname{In} \tau_{0}}{\sqrt{2} \sigma}\right)}
$$

For each class of cirrus, $\tau_{0}$ and $\sigma_{0}$ are deduced from lidar observations (Table 1). For clouds with optical depth $\leq 0.1$, the multiple scattering is negligible. ${ }^{44}$ The lidar observations of thin clouds C1, C2" , and C3 (Fig. 5) reveal a large part of the distribution of optical depths smaller than this value $(0.1)$. However, the $\mathrm{C} 2{ }^{\prime}$ class [Fig. 5(b)] reveals a significant fraction of optical depths larger than 0.1 .

We have calculated $\bar{\tau}$ with $\tau_{f}=0.1$ for each class (Table 2) to obtain the single scattering contribution of the cirrus particles at solar wavelengths. We have also calculated $\bar{\tau}$ using the true values of $\tau_{f}$ (Table 3) given by the observations (Fig. 5) for having realistic estimates taking into account multiple scattering. By comparing the results obtained with the two different values of $\tau_{f}$ for all classes of cirrus, we deduce an estimate of the net scattering effect of the solar light due to the cirrus nonhomogeneity.

Distributions of optical depth derived from lidar data show that the maximum value of the optical depth of $\mathrm{C} 1, \mathrm{C} 2{ }^{\prime \prime}$ and $\mathrm{C} 3$ cirrus is $\tau_{f}=0.562, \tau_{f}=0.1$, and $\tau_{f}=0.316$, respectively.

For thin clouds, even if we consider only single scattering effects, more visible light is received at ground for clouds with a nonhomogeneous distribution compared with homogeneous ones by $5 \%, 25 \%$, and $59 \%$ for $\mathrm{C} 1, \mathrm{C} 2{ }^{\prime \prime}$, and C3 (Table 2), respectively. When single and multiple scattering processes are applied $\left(\tau_{f}>0.1\right)$, the values of the ratio $\bar{\tau} / \tau_{0}$ are larger than values when only single scattering takes place $\left(\tau_{f}=0.1\right)$. With respect to the optical depth $\tau_{0}$, the scattering effect has increased by $65 \%$ and $88 \%$ for classes 1 and 3, respectively (Table 3 ). For $\mathrm{C} 22^{\prime \prime}$ class, the ratio $\bar{\tau} / \tau_{0}$ does not increase, given that $\tau_{f}=0.1$.

Table 2 Most frequently observed optical depth (second column) and mean optical depth of inhomogeneous clouds (third column) in case of single scattering processes (fourth column) as a function of cirrus class. Fifth column provides the ratio between the mean and the most frequently observed optical depth.

\begin{tabular}{lcccr}
\hline \hline Class of cirrus & $\tau_{0}$ & $\bar{\tau}$ & $\tau_{f}$ & $\bar{\tau} / \tau_{0}$ \\
\hline C1 & 0.032 & 0.033 & 0.1 & 1.05 \\
C2 $^{\prime}$ & 0.126 & 0.054 & 0.1 & 0.43 \\
C2 $^{\prime \prime}$ & 0.016 & 0.020 & 0.1 & 1.25 \\
C3 & 0.01 & 0.016 & 0.1 & 1.59 \\
\hline \hline
\end{tabular}

Table 3 Most frequently observed optical depth (second column) and mean optical depth of inhomogeneous clouds (third column) in case of maximum optical depth (fourth column) suggesting multiple scattering processes, as a function of cirrus class. Fifth column provides the ratio between the mean and the most frequently observed optical depth.

\begin{tabular}{lcccc}
\hline \hline Class of cirrus & $\tau_{0}$ & $\bar{\tau}$ & $\tau_{f}$ & $\bar{\tau} / \tau_{0}$ \\
\hline C1 & 0.032 & 0.052 & 0.562 & 1.65 \\
C2' & 0.126 & 0.203 & 1.78 & 1.61 \\
C2 11 & 0.020 & 0.1 & 1.25 \\
C3 & 0.016 & 0.019 & 0.316 & 1.88 \\
\hline \hline
\end{tabular}


As the mean geometrical depth of cirrus of classes $\mathrm{C} 1$ and $\mathrm{C} 3$, which are small $(0.9 \mathrm{~km}$ see Table 1), and as the fluctuations of the geometrical thickness around its mean value remains smaller than $0.1 \mathrm{~km}$, then the physical properties and the size distribution of the cirrus particles should be the same within the whole cloud. Consequently, the distribution of the optical depth in $\mathrm{C} 1$ and $\mathrm{C} 3$ cirrus classes originates mainly from the variation of the concentration of particles.

For the class $\mathrm{C} 2$ ' of cirrus, we have calculated the optical depth by assuming that only singlescattering processes occur $\left(\tau_{f}=0.1\right)$. We obtain $\bar{\tau}<\tau_{0}$ as shown in Table 2 . This means that the greatest contribution to the optical depth originates from parts of the cloud whose optical depths are lower than $\tau_{0}$. On the contrary, the results obtained taking into account the true value of $\tau_{f}$ is such that $\bar{\tau}>\tau_{0}$ with $\bar{\tau}>0.1$ (Table 3 ). The single- and multiple-scattering contributions, which take place when $\tau_{f}>0.1$ are important: the ratio $\bar{\tau} / \tau_{0}$ is 3.7 times greater when $\tau_{f}=1.78$ than when $\tau_{f}=0.1$. This does not mean that the increase of ground irradiance reaches 3.7, as a large part of the solar light is scattered back to space.

\section{Discussions and Conclusions}

The analysis of the variability of the temporal optical depth of cirrus observed by lidar with a $160 \mathrm{~s}$ resolution allowed us to access to the temporal resolution that can be converted into horizontal variability of around $2-\mathrm{km}$ resolution when advection is assumed to be the main cause of the variability. For the three observed cirrus classes, the variability has been statistically modeled using a log-normal distribution. For both classes of thin clouds, a monomodal distribution with a log-normal function is an appropriate fit of the optical depth, whereas for thick clouds, two lognormal functions are required to model the optical depth. This may be linked to multiple freezing processes.

For $\mathrm{C} 1, \mathrm{C} 2{ }^{\prime \prime}$, and $\mathrm{C} 3$ classes of thin clouds, the optical depth distribution shows that single scattering processes dominate the interaction between solar light and cirrus particles. However for $\mathrm{C}^{\prime}$ clouds multiple scattering processes have to be taken into account. In case multiple scattering is considered, the nonhomogeneous nature of the cirrus clouds induces more scattering of visible solar light than for homogeneous clouds. This phenomenon is amplified for thick clouds. This can be partly due to multiple scattering processes.

The statistical modeling of the horizontal distribution of the optical depth can be helpful for downscaling issues as encountered in numerical climate models or when developing retrievals of passive measurements from space having large fields of view.

The next step of this study will consist of coupling lidar measurements with ground irradiance measurements as well as fisheye pictures to take into account the horizontal size of the clouds in addition of the subgrid variability.

\section{Acknowledgments}

Lidar activities at Observatory of Haute-Provence are supported by Institut des Sciences de l'Univers (INSU) and cirrus investigations were also supported by Centre National de l'Etude Spatiale (CNES) in the framework of the Calypso validation. Coralie Jeannot has participated in this work during her Master classes in Sciences de l'Univers, Environnement of Paris 6 University.

\section{References}

1. IPCC, "IPCC Special Report on Renewable Energy Sources and Climate Change Mitigation," in Prepared by Working Group III of the Intergovernmental Panel on Climate, C. O.Edenhofer et al., Eds., Cambridge University Press, Cambridge, United Kingdom and New York, NY (2011).

2. H. LeTreut and B. McAvaney, "Equilibrium climate change in response to a $\mathrm{CO} 2$ doubling: an intercomparison of AGCM simulations coupled to slab oceans," Technical Report, Institut Pierre Simon Laplace, p. 18 (2000). 
Keckhut et al.: Subgrid-scale cirrus observed by lidar at mid-latitude: variability effects...

3. R. Pincus et al., "Evaluating the present-day simulation of clouds, precipitation, and radiation in climate models," J. Geophys. Res., 113(D14), 209 (2008), http://dx.doi.org/ 10.1029/2007JD009334.

4. J.R. Dim et al., "Influence of inhomogeneous cloud fields on optical properties retrieved from satellite observation," J. Geophys. Res., 112(D13), D13202 (2007), http://dx.doi.org/ 10.1029/2006JD007891.

5. N. Montoux et al., "Isentropic modelling of a cirrus event observed in the mid-latitude upper troposphere and lower stratosphere," J. Geophys. Res., 115(D2), D02202 (2010), http://dx .doi.org/10.1029/2009JD011981.

6. G. G. Mace et al., "Evaluation of cirrus cloud properties derived from MODIS data using cloud properties derived from ground-based observations collected at the ARM SGP site," J. Appl. Meteorol., 44(2), 221-240 (2005), http://dx.doi.org/10.1175/JAM2193.1.

7. D. L. Wu et al., "Vertical distributions and relationships of cloud occurrence frequency as observed by MISR, AIRS, MODIS, OML, CALIPSO, and CloudSat," Geophys. Res. Lett. 36(9), 1-5 (2009), http://dx.doi.org/10.1029/2009GL037464.

8. K. Sassen and B. Cho, "Subvisual-thin cirrus lidar dataset for satellite verification and climatological research," J. Appl. Meteorol. 31(11), 1275-1285 (1992), http://dx.doi.org/ 10.1175/1520-0450(1992)031<1275:STCLDF>2.0.CO;2.

9. P. Wang et al., "A 6-year climatology of cloud occurrence frequency from Stratospheric Aerosol and Gas Experiment II observations (1985-1990)," J. Geophys. Res., 101(D23), 407-429 (1996), http://dx.doi.org/10.1029/96JD01780.

10. J. T. Wiensz et al., "Optical property retrievals of subvisual cirrus clouds from OSIRIS limbscatter measurements," Atmos. Meas. Tech. Discuss. 5(4), 5313-5355 (2012), http://dx.doi .org/10.5194/amtd-5-5313-2012.

11. G. Pappalardo et al., "EARLINET correlative measurements for CALIPSO: First intercomparison results," J. Geophys. Res. 115(D4), D00H19 (2010), http://dx.doi.org/10.1029/ 2009JD012147 [printed 116(D4), 2011].

12. J.-C. Dupont et al., "Macrophysical and optical properties of midlatitude clouds from four ground-based lidars and collocated caliop observations," J. Geophys. Res. 115(D4), D00H24 (2010), http://dx.doi.org/10.1029/2009JD011943.

13. G. Pretor-Pinney, International Cloud Atlas, Vol. I, Manual on the Observation of Clouds and Other Meteors, World Meteorological Organizaion (WMO), Geneva (1995).

14. H. Hildebrandson, "Remarks concerning the nomenclature of clouds for ordinary use," Q. J. Roy. Meteorol. Soc. 13(62), 140-146 (1887), http://dx.doi.org/10.1002/qj.4970136211.

15. K. Sassen, M. K. Griffin, and G. C. Dodd, "Optical scattering and microphysical properties of subvisible cirrus clouds, and climatic implications," J. Appl. Meteorol. 28(2), 91-98 (1989), http://dx.doi.org/10.1175/1520-0450(1989)028<0091:OSAMPO>2.0.CO;2.

16. J. Hogan, "Fast lidar and radar multiple-scattering models. Part I: small-angle scattering using the photon variance-covariance method," J. Atmos. Sci., 65(12), 3621-3635 (2008), http://dx.doi.org/10.1175/2008JAS2642.1.

17. A. Heymsfield and J. Iaquinta, "Cirrus crystal terminal velocities," J. Atmos. Sci. 57(7), 916-938 (2000), http://dx.doi.org/10.1175/1520-0469(2000)057<0916:CCTV>2.0.CO;2.

18. R. Pincus and S. Klein, "Unresolved spatial variability and microphysical process rates in large-scale models," J. Geophys. Res. 105(D22), 27059-27065 (2000), http://dx.doi.org/10 $.1029 / 2000 J D 900504$.

19. Y. Gu and K.N. Liou, "Radiation parameterization for three-dimensional inhomogeneous cirrus cloud: application to climate models," J. Clim. 14(11), 2443-2457 (2001), http://dx .doi.org/10.1175/1520-0442(2001)014<2443:RPFTDI>2.0.CO;2.

20. S. K. Cox, "Cirrus clouds and the climate," J. Atmos. Sci. 28(8), 1513-1515 (1971), http:// dx.doi.org/10.1175/1520-0469(1971)028<1513:CCATC >2.0.CO;2.

21. D. R. Dowling and L. F. Radke, "A summary of the physical properties of cirrus clouds," J. Appl. Meteorol. 29(9), 970-978 (1990), http://dx.doi.org/10.1175/1520-0450(1990) 029<0970:ASOTPP>2.0.CO;2.

22. J. Jumelet et al., "Statistical estimation of stratospheric particle size distribution by combining optical modeling and lidar scattering measurements," Atmos. Chem. Phys. 8(17), 54355448 (2008), http://dx.doi.org/10.5194/acp-8-5435-2008. 
Keckhut et al.: Subgrid-scale cirrus observed by lidar at mid-latitude: variability effects...

23. B. Carlin et al., "High-cloud horizontal inhomogeneity and solar albedo bias," J. Clim. 15(17), 2321-2339 (2002), http://dx.doi.org/10.1175/1520-0442(2002)015<2321: HCHIAS $>2.0 . \mathrm{CO} ; 2$.

24. B. Kärcher and J. Strom, "The roles of dynamical variability and aerosols in cirrus cloud formation," Atmos. Chem. Phys. 3(3), 823-838 (2003), http://dx.doi.org/10.5194/acp-3823-2003.

25. J. Kay, M. Backer, and D. Hegg, "Microphysical and dynamical controls on cirrus cloud optical depth distribution," J. Geophys. Res. 111(D24), D24205 (2006), http://dx.doi.org/10 .1029/2005JD006916.

26. L. Goldfarb et al., "Cirrus climatological results from lidar measurements at OHP

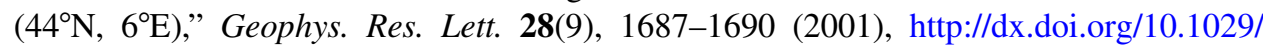
2000GL012701.

27. P. Keckhut et al., "Cirrus classification at mid-latitude from systematic lidar observations," J. Appl. Meteorol. Climatol. 45(2), 249-258 (2006), http://dx.doi.org/10.1175/ JAM2348.1

28. P. Keckhut et al., "Indications of thin cirrus clouds in the stratosphere at mid-latitudes," Atmos. Chem. Phys. 5(12), 3407-3414 (2005), http://dx.doi.org/10.5194/acp-5-3407-2005.

29. A. Hauchecorne and M.-L. Chanin, "Density and temperature profiles obtained by lidar between 30 and 70 km," Geophys. Res. Lett. 7(8), 564-568 (1980), http://dx.doi.org/ 10.1029/GL007i008p00565.

30. P. Chazette et al., "Study of the optical, geometrical and dynamical properties of stratospheric post-volcanic aerosols from lidar remote sensing at $532 \mathrm{~nm}$, following the eruptions of El Chichon and Mount Pinatubo,” J. Geophys. Res. 100(D11), 23195-23207 (1995), http://dx.doi.org/10.1029/95JD02268.

31. P. Keckhut, A. Hauchecorne, and M. L. Chanin, "A critical review of the database acquired for the long term surveillance of the middle atmosphere by the French Rayleigh lidar," J. Atmos. Oceanic Technol. 10(6), 850-867 (1993), http://dx.doi.org/10.1175/1520-0426 (1993)010<0850:ACROTD>2.0.CO;2.

32. C. M. R. Platt, "Lidar observation of a mixed-phase Altostratus cloud," J. Appl. Meteorol. 16(4), 339-345 (1977), http://dx.doi.org/10.1175/1520-0450(1977)016<0339: LOOAMP>2.0.CO;2.

33. S. A. Young et al., "Optical properties and phase of some midlatitude, midlevel clouds in ECLIPS," J. Appl. Meteorol. 39(2), 135-153 (2000), http://dx.doi.org/10.1175/1520-0450 (2000)039<0135:OPAPOS>2.0.CO;2.

34. C. D. Westbrook et al., "Doppler lidar measurements of oriented planar ice crystals faling from supercooled and glaciated layer clouds," Q. J. R. Meteorol. Soc. 36(12), $1-19$ (2009).

35. A. Ansmann et al., "Independent measurement of extinction and backscatter profiles in cirrus clouds by using a combined Raman elastic-backscatter lidar," Appl. Opt. 31(33), 7113-7113 (1992), http://dx.doi.org/10.1364/AO.31.007113.

36. B. Cadet et al., "Improved retrievals of the optical properties of cirrus clouds by a combination of lidar methods," Appl. Opt. 44(9), 1726-1734 (2005), http://dx.doi.org/10.1364/ AO.44.001726.

37. C. M. R. Platt and A. C. Dilley, "Determination of the cirrus particle single-scattering phase function from lidar and solar radiometric data," Appl. Opt. 23(3), 380-386 (1984), http://dx.doi.org/10.1364/AO.23.000380.

38. K. Sassen and J. M. Comstock, "A midlatitude cirrus cloud climatology from the facility for atmospheric remote sensing. Part III: radiative properties," J. Atmos. Sci. 58(15), 2113-2127 (2001), http://dx.doi.org/10.1175/1520-0469(2001)058<2113:AMCCCF>2.0 .CO;2.

39. E. Giannakaki et al., "Optical and geometrical characteristics of cirrus clouds over a midlatitude lidar station," Atmos. Chem. Phys. 7(4), 5519-5530 (2007), http://dx.doi.org/10 $.5194 /$ acp-7-5519-2007.

40. W. N. Chen, C. W. Chiang, and J. B. Nee, "The lidar ratio and depolarization ratio for cirrus clouds," Appl. Opt. 41(30), 6470-6476 (2002), http://dx.doi.org/10.1364/ AO.41.006470. 
41. J. P. Vernier et al., "Tropical stratospheric aerosol layer from CALIPSO lidar observations," J. Geophys. Res. 114(D4), D00H10 (2009), http://dx.doi.org/10.1029/2009JD011946.

42. N. Lamquin et al., "A global climatology of upper-tropospheric ice supersaturation occurrence inferred from the Atmopheric Infrared Sounder calibrated by MOZAIC," Atmos. Chem. Phys. 12(1), 381-405 (2012), http://dx.doi.org/10.5194/acp-12-381-2012.

43. J.H. Seinfeld and S.N. Pandis, "Atmospheric chemistry and physics, from air pollution to climate change," J. Atmos. Chem. 37(2), 212-214 (2000), http://dx.doi.org/10.1023/A: 1006483708571.

44. H. C. Van de Hulst, Light Scattering by Small Particles, John Wiley \& Sons, Inc., New York (1957).

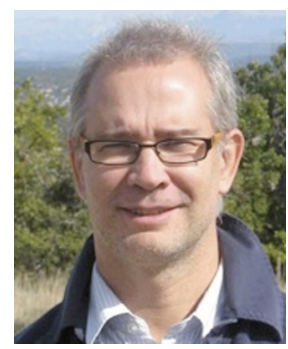

Philippe Keckhut is a senior researcher at Laboratoire Atmosphères, Milieux, Observations Spatiales, vice director of Institut Pierre Simon Laplace. He get his PhD degree in 1991 at Paris 6 University and was a visiting scientist at NOAA (Washington DC) in 1994 through a grant from the National Research Council. He has 25 years of research experience in the field of lidar instrumentation and atmospheric climatology, and is the author of 103 publications in peer review literature ( $\mathrm{H}$ factor: 26$)$. $\mathrm{He}$ is in charge of routine lidar measurements in operation since 1979. He was awarded for Louis Armand price from the French Academy (1992) and Norbert-Gerbier Mumn prices from the World Meteorological Organization (2003 and 2005). He was deputy director of the FP6 Geomon project (2007 to 2011). He was a member of the Stratospheric Temperature Trends Assessment (SPARC/WCRP), International Coordination-group on Laser Atmospheric Studies (ICLAS), Atmospheric Chemistry Validation Team ENVISAT/ESA and Mesospheric Temperature Trend Assessment (IAGA/ ICMA/PS-MOS) expert groups.

Biography and photograph of the authors are not available. 